\title{
Recovery of human gut microbiota genomes with third-generation sequencing
}

\author{
Yanfei Li ${ }^{1,2}$, Yueling Jin', Jianming Zhang ${ }^{3}$, Haoying Pan ${ }^{1}$, Lan $\mathrm{Wu}^{1,2}$, Dingsheng Liu', Jinlong Liu (1)', Jing Hu (i) ${ }^{4}$ and \\ Junwei Shen (1) $^{5}$
}

\begin{abstract}
Human gut microbiota modulates normal physiological functions, such as maintenance of barrier homeostasis and modulation of metabolism, as well as various chronic diseases including type 2 diabetes and gastrointestinal cancer. Despite decades of research, the composition of the gut microbiota remains poorly understood. Here, we established an effective extraction method to obtain high quality gut microbiota genomes, and analyzed them with thirdgeneration sequencing technology. We acquired a large quantity of data from each sample and assembled large numbers of reliable contigs. With this approach, we constructed tens of completed bacterial genomes in which there were several new bacteria species. We also identified a new conditional pathogen, Enterococcus tongjius, which is a member of Enterococci. This work provided a novel and reliable approach to recover gut microbiota genomes, facilitating the discovery of new bacteria species and furthering our understanding of the microbiome that underlies human health and diseases.
\end{abstract}

\section{Introduction}

There is increasing evidence that the gut microbiota, the human commensals, influences normal physiological functions, such as maintenance of barrier homeostasis and modulation of metabolism, inflammation, immunity, and development ${ }^{1-3}$. Further, disorder of the gut microbiota is a potential risk factor for various chronic diseases such as type 2 diabetes, gastrointestinal cancer, and brain disorders $^{4-6}$. Furthermore, the gut microbiota influences drug effectiveness because of its effects on drug metabolism $^{7,8}$. Despite decades of research, the composition of the gut microbiota remains unidentified. For example, through analysis of more than 150,000 human microbial genomes, Edoardo et al. recapitulated nearly 5000 species-level

\footnotetext{
Correspondence: Jing Hu (hujing06@tongji.edu.cn) or

Junwei Shen (shenjunwei@tongji.edn.cn)

'Shanghai University of Medicine \& Health Sciences affiliated Zhoupu Hospital, 201318 Shanghai, China

${ }^{2}$ School of Basic Medical Sciences and Shanghai Key Laboratory of Molecular Imaging, Shanghai University of Medicine and Health Sciences, 201318

Shanghai, China

Full list of author information is available at the end of the article

Edited by H.U. Simon
}

genome bins, in which $77 \%$ are not in public repositories ${ }^{9}$. One of the main difficulties is that many microbes are uncultivable and require isolation ${ }^{9}$. In addition, culturedependent genomic research is rare in large cohorts ${ }^{10}$. Bypassing the need to cultivate microbes, high-throughput sequencing overcomes these shortages and is a powerful method for bacterial metagenomics.

There are two main methods for bacteria metagenome sequencing: 16S rRNA-based sequencing and wholemetagenome shotgun sequencing (WGS). The 16S rRNA-based sequencing is widely used to assess microbial communities due to its low cost, time efficiency, and ability to provide a full overview of the community ${ }^{11,12}$. However, as only a single region of 16s rRNA in the genome is detected, it provides limited information about the microbial community ${ }^{13,14}$. Furthermore, it underestimates the diversity of microbes. Compared with $16 \mathrm{~s}$ rRNA sequencing, WGS technology examines whole bacterial genomes, and provides more accurate detection at the species and diversity levels ${ }^{15}$. Through WGS technology, it is feasible to assemble the entire bacterial metagenome. However, there are several drawbacks in

\section{(c) The Author(s) 2021}

(c) (i) Open Access This article is licensed under a Creative Commons Attribution 4.0 International License, which permits use, sharing, adaptation, distribution and reproduction cc) in any medium or format, as long as you give appropriate credit to the original author(s) and the source, provide a link to the Creative Commons license, and indicate if changes were made. The images or other third party material in this article are included in the article's Creative Commons license, unless indicated otherwise in a credit line to the material. If material is not included in the article's Creative Commons license and your intended use is not permitted by statutory regulation or exceeds the permitted use, you will need to obtain permission directly from the copyright holder. To view a copy of this license, visit http://creativecommons.org/licenses/by/4.0/. 
WGS technology owing to short sequencing reads (mostly $<200 \mathrm{bp}$ ). For example, many microbiota genomes are fragmented into numbers of contigs ${ }^{16}$. Besides, it is difficult to assemble the de novo genome because there is too little sequence information ${ }^{16,17}$. Moreover, great phenotypic differences exist even between highly related strains of the same species ${ }^{18,19}$, but the differences between these strains are difficult to distinguish by WGS. Therefore, there is a dire need to detect the bacterial metagenome through more powerful sequencing methods.

Third-generation sequencing (TGS) technology, also known as long-read sequencing, detects the isolated genomic DNA without amplification and produces surprising long reads (average $10-20 \mathrm{~kb})^{20}$. Compared with the second-generation sequencing technology of 16s rRNA and WGS, it can detect much longer fragments, so TGS produces genome assemblies of unprecedented quality ${ }^{20}$. For example, Hui et al. de novo assembled a chromosomelevel reference genome of the red-spotted grouper with TGS $^{21}$. Though TGS has been used in eukaryotic genome detection extensively, but unfortunately its application in microbiota genomes have not been of sufficient scale. For instance, Johanna et al. assembled a certain specific microbial species living in the vagina with an abundance of more than $75 \%{ }^{22}$. Thidathip et al. compared the taxonomic abundance of gut microbiota of head and neck cancer patients; while the mean length is approximately $1 \mathrm{~kb}$, which is only a tenth of that of the buffalo genome $(11.5 \mathrm{~kb})^{23,24}$. One difficulty in the detection of gut microbiota genomes is that it is challenging to obtain high quality genomic DNA, because of complex fecal features. Therefore, large numbers of bacterial species in the gut microbiota have not yet been identified. In this study, we successfully extracted high quality gut microbial genomic DNA. By detecting the gut microbiota metagenome via Single Molecule Real-Time (SMRT) Sequencing of Pacbio, we assembled the bacteria genomes efficiently and discovered new species of bacteria.

\section{Materials and methods}

\section{Sample collection and bacterial genomic DNA extraction}

All methods in this study were approved by the Research Medical Ethics Committee of Shanghai University of Medicine \& Health Sciences affiliated Zhoupu Hospital. Fecal samples were collected from a 34-year-old male and a 10-month-old baby and stored in an ultra-low temperature freezer (Haier, Qingdao, China). Genomic DNA extraction was performed with a FastDNA Spin Kit for Feces (MP Biomedicals, Irvine, CA, USA). To acquire sufficient high-quality gut microbiota genomic DNA, we improved the experimental procedure as follows: we added $500 \mathrm{mg}$ feces in a $2 \mathrm{~mL}$ Lysing Matrix E tube, mixed the feces with $825 \mu \mathrm{L}$ sodium phosphate buffer and $275 \mu \mathrm{L}$ PLS solution, and shook the mix and vibrated for
$15 \mathrm{~s}$. Then, we centrifuged the samples at $14,000 \times g$ for $5 \mathrm{~min}$ at room temperature and decanted the supernatant. Subsequently, we added $978 \mu \mathrm{L}$ sodium phosphate buffer, shook the mix, and vibrated the mixture for $15 \mathrm{~s}$, then added $122 \mu \mathrm{L}$ MT Buffer and shook up and down gently for $5 \mathrm{~min}$. Then, we placed the samples in the shaker at $4{ }^{\circ} \mathrm{C}$ for $30 \mathrm{~min}$ followed by centrifugation at $14,000 \times g$ for $5 \mathrm{~min}$ and transferred the supernatant to a clean EP tube. Then, $250 \mu \mathrm{L}$ of PPS solution was added and the samples were vigorously mixed and incubated at $4{ }^{\circ} \mathrm{C}$ for $10 \mathrm{~min}$ followed by centrifugation at $14,000 \times g$ for $2 \mathrm{~min}$. The supernatant was transferred to the binding matrix solution in a $15 \mathrm{~mL}$ conical tube and shook gently for $5 \mathrm{~min}$. Then, the samples were centrifuged at $14,000 \times g$ for $2 \mathrm{~min}$ and the supernatant was decanted. Afterwards, we washed the binding mixture pellet with $1 \mathrm{~mL}$ wash buffer $\# 1$ and transferred the binding mixture to a SPIN filter tube and centrifuged at $14,000 \times g$ for $1 \mathrm{~min}$. We emptied the catch tube and added $500 \mu \mathrm{L}$ of prepared wash buffer \#2 to the SPIN filter tube and gently resuspended the pellet. Afterwards, we centrifuged the samples at $14,000 \times g$ twice to extract residual ethanol. Finally, we transferred the SPIN filter bucket to a clean $1.9 \mathrm{~mL}$ catch tube and added $100 \mu \mathrm{L}$ TES to resuspend the genomic DNA. DNA was detected using agarose gel electrophoresis, and the top bands were isolated and purified with a DNA Purification Kit (Finegene, Shanghai, China). The DNA concentration and integrity were assessed using a NanoDrop2000 spectrophotometer (Thermo Fisher Scientific, Waltham, MA, USA).

\section{Library construction}

For PacBio sequencing library preparation and SMRT sequencing, DNA was fragmented by a Covaris g-TUBE device $(10 \mathrm{~kb})$ and was concentrated with AMPure PB beads according to the manufacturer's instructions (Beckman Coulter Co., Brea, CA, USA). DNA damage and ends were repaired in a LoBind microcentrifuge tube. Blunt ligation reaction was performed by adding $1 \mu \mathrm{L}$ of blunt adapter $(20 \mu \mathrm{M})$ and $1 \mu \mathrm{L}$ of ligase to $30 \mu \mathrm{L}$ DNA followed by incubation at room temperature for $15 \mathrm{~min}$. SMRTbell $^{\mathrm{TM}}$ templates were purified with AMPure $\mathrm{PB}$ beads and the concentration was measured by Qubit. Sequencing was performed on a PacBio Sequel instrument by OE Biotech Co., Ltd. (Shanghai, China).

\section{Bioinformatics analysis}

Metagenome assembly was performed with flye software after obtaining valid reads. Open reading frame prediction of assembled scaffolds using prodigal was performed and translated into amino acid sequences. Non-redundant gene sets were built for all predicted genes using CD-HIT. The clustering parameters were $95 \%$ identity and $90 \%$ coverage. The longest gene was selected 
as the representative sequence of each gene set. The gene set representative sequence (amino acid sequence) was annotated with NR, KEGG, COG, SWISSPROT, and GO databases with an e-value of $1 \mathrm{e}^{-5}$. The taxonomy of the species was obtained as a result of the corresponding taxonomy database of the NR library.

\section{$\mathrm{NCBI}$ prokaryote genome databases}

Prokaryotic genome data were acquired from databases in NCBI (https://www.ncbi.nlm.nih.gov/genome/ browse\#!/prokaryotes/). A total of 266,319 prokaryote genomes have been identified until now [chromosome (3186), complete $(19,702)$, contig $(141,127)$, scaffold $(102,304)$ ] (Supplementary Fig. 1). A total of 108,506 prokaryote genomes were associated with humans [chromosome (1539), complete $(8179)$, contig $(57,034)$, scaffold $(41,754)]$ (Fig. 1a).

\section{NCBI blast}

We acquired the full-length 16s rRNAs from the assembled contigs and blasted the 16s rRNAs using the NCBI database (https://blast.ncbi.nlm.nih.gov/Blast. cgi?PROGRAM=blastn\&PAGE_TYPE $=$ BlastSearch\&\&LINK_LOC=blasthome). Then, we screened similar sequences in the order of identity, and downloaded the relative sequences. We identified the bacterial species of the assembled contigs and analyzed their evolutionary relationship.

\section{ClustalW and phylogenetic tree analysis}

To examine the differences between full-length $16 \mathrm{~s}$ rRNAs, we compared the 16s rRNAs and visualized the differences using BioEdit software (Borland Software Corporation, Scotts Valley, CA, USA). For phylogenetic tree analysis, we blasted the full-length 16s rRNAs in the NCBI database (https://blast.ncbi.nlm.nih.gov/Blast.cgi?P ROGRAM=blastn\&PAGE_TYPE $=$ BlastSearch\&LINK_LOC = blasthome) and selected the most related full-length $16 \mathrm{~s}$ rRNAs by identity. With these 16s rRNAs, we analyzed the phylogenetic tree with $\mathrm{Mega}^{25}$. The average nucleotide identity (ANI) were used to analyze whether two genomes belong to the same species ${ }^{26}$.

\section{Single bacteria analysis}

Single bacteria analyses included gene prediction, noncoding RNA (ncRNA) prediction, repeat sequence prediction, nonredundant analysis, and common function potential analyses. We performed gene prediction with the Prokaryotic Dynamic Programming Genefinding Algorithm [prodigal (v2.6.3)] ${ }^{27}$; the results included gene number, average gene length (bp), and GC\% (gene region). The ncRNA predictions were harnessed using tRNAscan-SE $\quad(\mathrm{v} 1.3 .1)^{28}$ (tRNA), RNAmmer $\quad(\mathrm{v} 1.2)^{29}$ (rRNA), and Rfam(v10.0) ${ }^{30}$ (sRNA). Repeat sequence prediction was analyzed with RepeatMasker $(\mathrm{v} 4.0 .7)^{31}$. Common function potential analyses were performed using the Non-Redundant (https://www.ncbi.nlm.nih. gov), Swissprot (http://www.uniprot.org), KEGG (http:// www.genome.jp/kegg/pathway.htmL), Cluster of Orthologous Groups of proteins (https://www.ncbi.nlm.nih.gov/ COG/), comprehensive antibiotic resistance database (CARD) (https://card.mcmaster.ca) ${ }^{32}$, and carbohydrateactive enzymes (http://www.cazy.org) databases ${ }^{33}$.

\section{Statistical analysis}

$\mathrm{R}$ programming language version 3.4.3 was used for statistical analysis. Statistical significance between two groups was determined using an unpaired two-tailed Student's $t$-test. Data are presented as mean \pm SD (standard deviation) or mean \pm SEM (standard error of the mean) as indicated in the figure legends. $P$-values were considered statistically significant at $P<0.05$.

\section{Results}

\section{The characteristics of gut microbiota genomes}

We analyzed the data of prokaryotes genomes in the NCBI database and found that complete genomes account for only a small portion (https://www.ncbi.nlm.nih.gov/ genome/browse\#!/prokaryotes/) (Fig. 1a and Supplementary Fig. 1a), consistent with previous research ${ }^{9}$. To assemble de novo complete genomes of gut microbes, we detected the gut microbiota metagenome using TGS technology. To obtain high quality gut microbiota genome samples for TGS, we attempted to improve the integrity and quantity of genome DNA. Initially, the isolated genomic DNA was short. After trying many different kits, we acquired fairly complete bacteria genomes. Then, we tried to improve the yield of genomic DNA. We isolated and purified the gut microbiota genome DNA with a DNA purification kit. Finally, we acquired two high quality samples: a 34-year-old male and a 10-monthold baby.

We detected the whole metagenome of the two samples using TGS technology. We acquired 91.7 and $81.7 \mathrm{~Gb}$ data from the baby and adult sample, respectively. Through analysis of genome databases in NCBI, we found that most of the bacterial genomes are larger than $0.5 \mathrm{Mb}$ and the smallest bacteria genome was approximately $0.1 \mathrm{Mb}$ (Supplementary Table 1 and Supplementary Fig. 1b). Therefore, we chose 0.1 and $0.5 \mathrm{Mb}$ as cutoff values to analyze the assembly contigs (Fig. 1b). In the baby sample, there were 43 contigs larger than $0.5 \mathrm{Mb}$ and 248 contigs larger than $0.1 \mathrm{Mb}$. Similarly, in the adult sample, there were 33 contigs larger than $0.5 \mathrm{Mb}$ and 237 contigs larger than $0.1 \mathrm{Mb}$ (Fig. 1b). We analyzed the 16s rRNA sequences from the longest contig (Contig_511). The six $16 \mathrm{~S}$ rRNA sequences of contig 511 were almost the same, with only two different bases in the over $1.5 \mathrm{~kb}$ sequence 


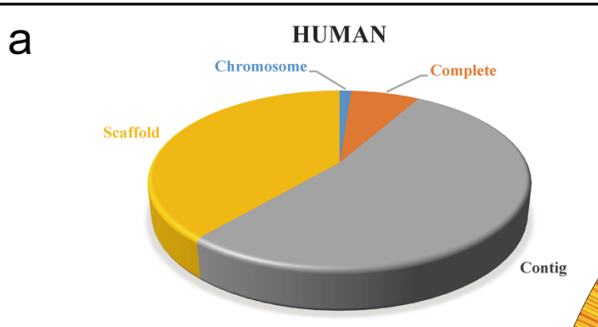

C

b
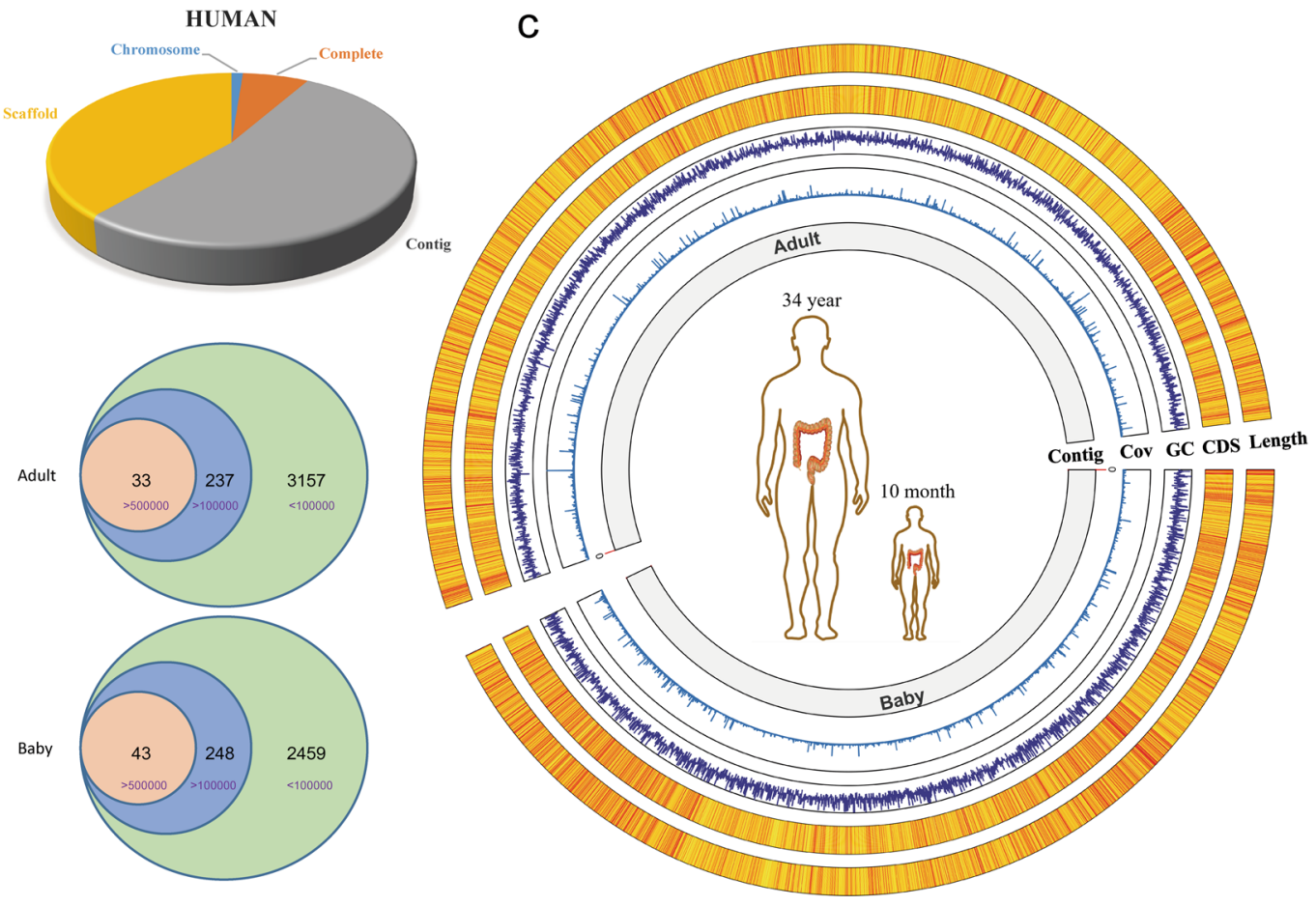

d

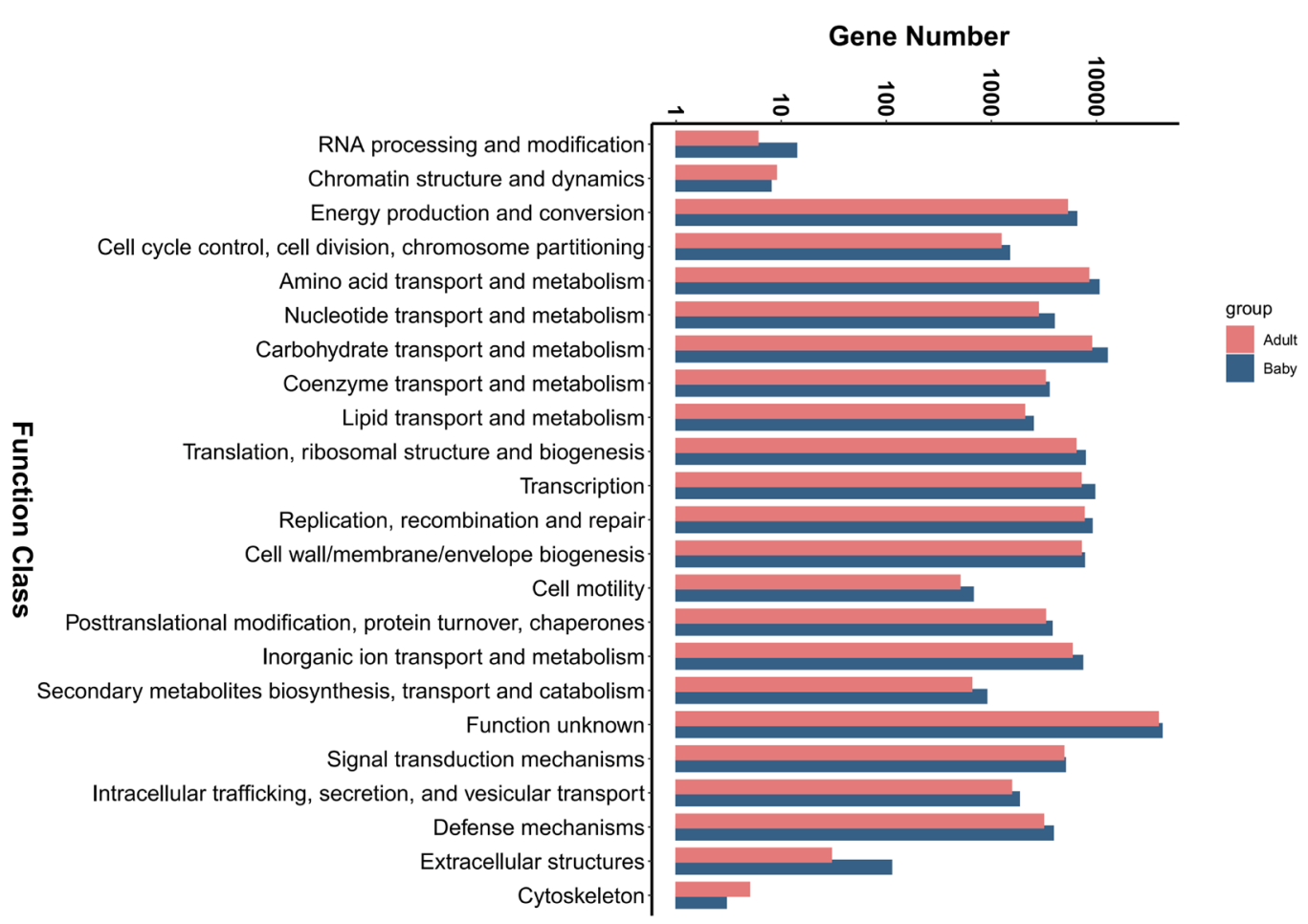

Fig. 1 The characteristics of the two samples. a Pie chart comprising four classes of human bacteria sequence data in the NCBI databases: chromosome, complete, scaffold, and Contig. b Numbers of contigs with different lengthes acquired from the two samples, relative to the adult sample (up) and the baby sample (down). c The whole characteristics of the two samples. The corresponding contig length and CDS number were depicted in the first outer layer and the second outer layer, respectively; the GC content was depicted in the third outer layer; the contig number and the coverage were depicted in the first inner layer and the second inner layer, respectively. $\mathbf{d}$ Function analyses of all the CDSs of the two samples. 
(Supplementary Fig. 1c). Interestingly, the $16 \mathrm{~S}$ sequence in contig_511 had fewer differences than that in Escherichia coli (U00096.3) (Supplementary Fig. 1c). This result suggested that the methods we used were reliable (Supplementary Fig. 1c). Moreover, the contig length was significantly related to the number of coding sequences (CDSs) (Fig. 1c). Through analysis of the functional potential of CDSs, we found that many CDSs were correlated with the metabolism of amino acids and carbohydrates. Interestingly, the functional potential of a large number of genes was unknown, indicating that there are still numerous valuable genes worth investigating in gut microbes (Fig. 1d).

\section{Aspects of the contigs in the two samples}

Because most bacterial genomes are greater than $0.5 \mathrm{Mb}$, we further analyzed the contigs greater than $0.5 \mathrm{Mb}$ in the two samples (Supplementary Table 2). Among these contigs, 10 were circular (Fig. 2a) and 12 were more than $99 \%$ complete (Fig. 2b). Nine contigs were both circular and complete (>99\%) (Fig. 2c). Therefore, we focused on these nine contigs and analyzed their features. The full-length 16S rRNA blast analyses showed that less than $50 \%$ (four of nine contigs) of these contigs could be completely matched with existing sequences in the NCBI databases (Fig. 2d, e; indicated by red arrow). Five were not completely matched, indicating they might be new species or subspecies (Fig. 2d, e). To investigate the taxonomic location of the nine contigs, we analyzed them by phylogenetic analysis using full-length 16S rRNA. By comparison with common bacteria such as Bacterioides fragilis and E. coli, we successfully identified their taxonomic location in the phylogenetic tree (Fig. 2e).

\section{Genomic characteristics of the five non-matched genomes}

We further analyzed the five genomes that could not be completely matched. Their genome lengths ranged from 1.5 to $3.5 \mathrm{Mb}$ (Supplementary Table 3 and Fig. 3a). Despite the large difference in genome length, the average length of each gene was similar (Fig. 3a). In contrast, the difference in GC content between these genomes was large (Fig. $3 \mathrm{~b}$ ). We also analyzed some special structures of the genomes, including ncRNA and repeat sequences. Interestingly, the number of rRNAs and tRNAs in each genome was correlated (Fig. 3c). Each genome had all types of repeat sequences; simple repeats were the major repeat type (Fig. 3d). Intriguingly, we found that all five bacteria exhibited streptomycin resistance, possibly a result of long-term antibiotic use (Fig. 3e) and suggesting that the extensive use of antibiotics may have irreversibly transformed the human gut microbiome. To determine the classification to which they belong, we blasted the full-length $16 \mathrm{~S}$ rRNAs in NCBI databases (https://blast.ncbi.nlm.nih.gov/Blast.
cgi?PROGRAM=blastn\&PAGE_TYPE $=$ BlastSearch\&LLINK_LOC=blasthome). Four contigs (contig_82, contig_242, contig_318, and contig_511) had high identities (>99\%), while the identity of contig_638 was less than 97\% (Fig. 3f).

\section{Phylogeny of four high identity non-matched genomes}

We then analyzed the bacteria with identities of more than $99 \%$, and detected their accurate taxonomic location by phylogenetic tree analysis. We blasted the full-length $16 \mathrm{~s}$ rRNAs of these genomes with the NCBI databases, and selected the most related species (rank by identity) for further analysis. Contig_82 belongs to Collinsella erofaciens and might be a new subspecies (Fig. 4a). Similarly, contig_242 is a subspecies of Megasphaera micronuciforrnis, contig_318 is a subspecies of Lactobacillus dextrinicus, and contig_511 is a subspecies of Lactobacillus smilis (Fig. 4b-d). Analysis of non-redundant database annotations confirmed these results (Supplementary Fig. 3a-d). We then compared the full-length $16 \mathrm{~s}$ rRNAs of these genomes with those of related bacterial strains, and found that the differences between them were small (Fig. 4e). The most considerable difference in contig_318 was only five bases among more than 1500 bases (Fig. 4e). Interestingly, Megasphaera micronuciforrnis (contig_242) was a conditional pathogen; therefore we sought to evaluate whether healthy people also carry other conditional or common pathogens. We selected 27 common and conditional pathogens, including E. coli, Staphylococcus aureus, Shigella flexneri, and Helicobacter pylori. Only S. flexneri was found in the gut of the adult and baby samples, while other pathogenic bacteria were not found (Fig. 4f).

\section{Genomic aspects of the new species Enterococcus tongjius}

We analyzed contig_638, which showed poor identity. Through the analysis of non-redundant database annotations, we found it difficult to find a bacterium to match contig_638, because the most similar Enterococcus faecalis showed only $8 \%$ similarity (Fig. $5 \mathrm{a}$ ). We sought to determine its classification through NCBI blast and 16s rRNA phylogenetic tree analysis. Excitingly, we discovered that this bacterium belongs to Enterococcus (Supplementary Fig. 4a). Compared with other similar bacteria Enterococcus saccharolyticus and Enterococcus faecalis, we found that there were considerable differences in the 16s rRNA sequences (Supplementary Fig. 4b). To confirm whether Enterococcus tongjius is a new species, we compared its genome with some similar Enterococci genomes (Fig. 5b). The ANI results showed that it was a total new species since the ANI score was much lower than 0.9 (Fig. 5c). Besides, the flower-plot showed that though Enterococcus tongjius shared only 867 core genes with other Enterococci; it had 1162 distinctive genes (Fig. 5d). Because it was found 


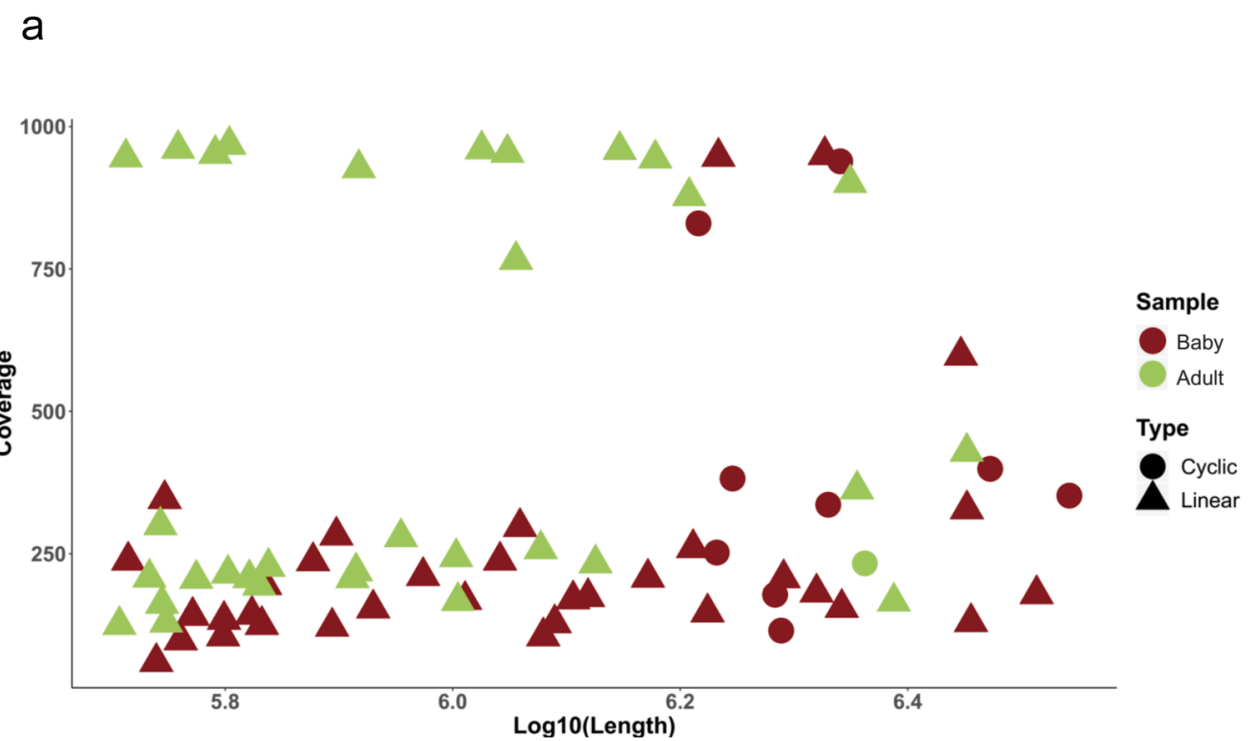

b

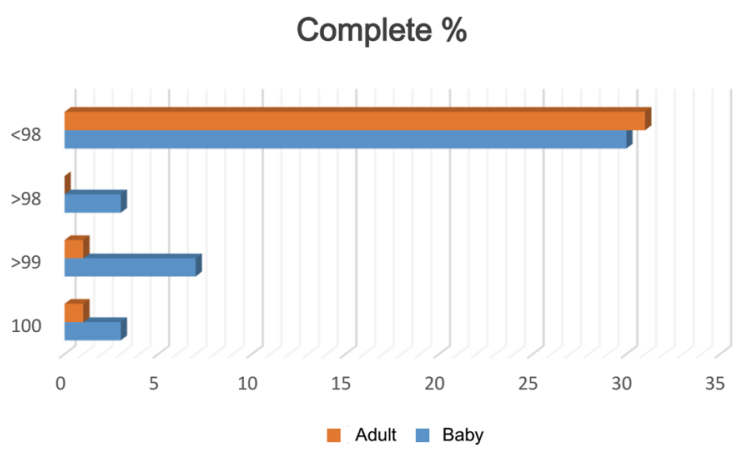

d

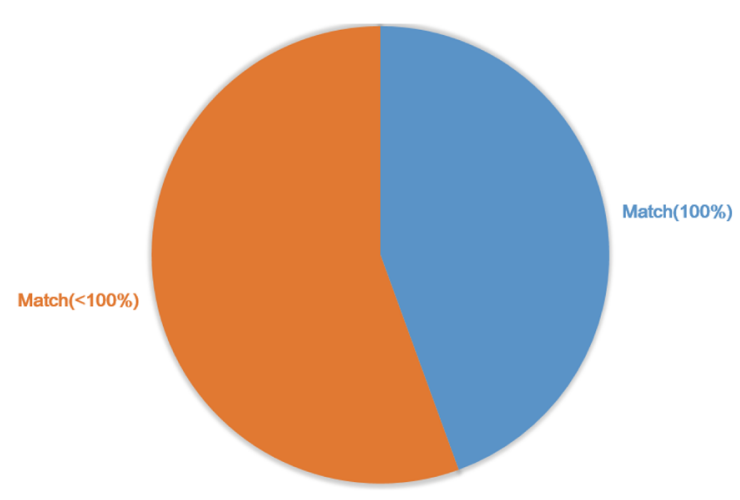

C

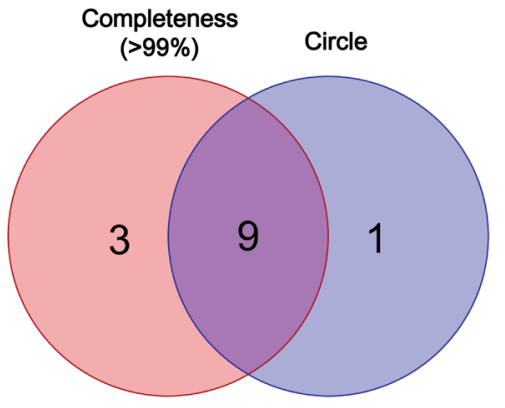

\footnotetext{
Fig. 2 The different aspects of the contigs (longer than $0.5 \mathrm{Mb}$ ) in the two samples. a The length and coverage of the contigs in the two samples (longer than $0.5 \mathrm{Mb}$ ). b Complete analysis of the contigs in the two samples. c Wenn diagram shows the number of contigs (Complete $\geq 99 \%$ or circle). $\mathbf{d}$ Pie diagram shows the number of the nine genomes whether they match public genomes. e Phylogenetic tree of the nine assembled genomes, each arrow shows the completely matched contig.
} 
a

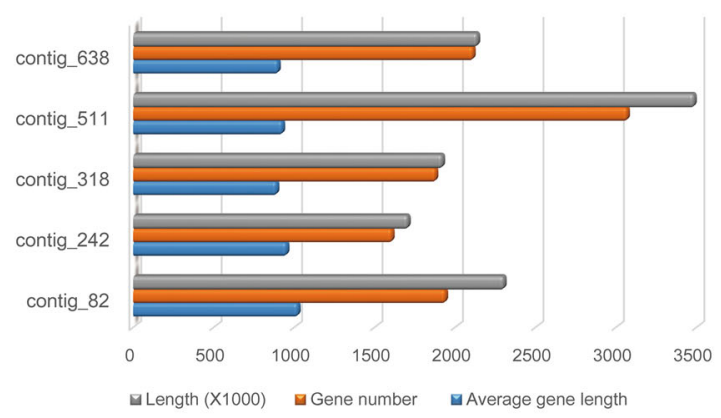

C

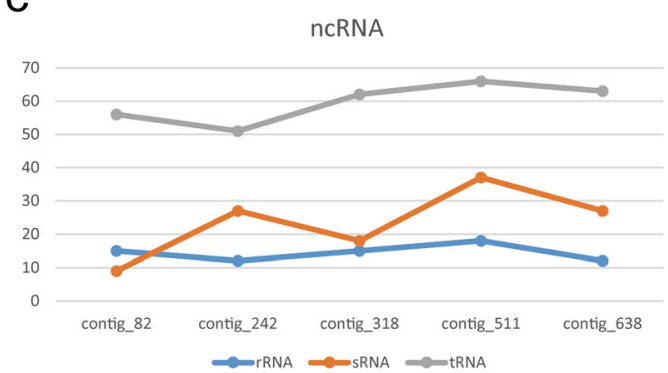

e

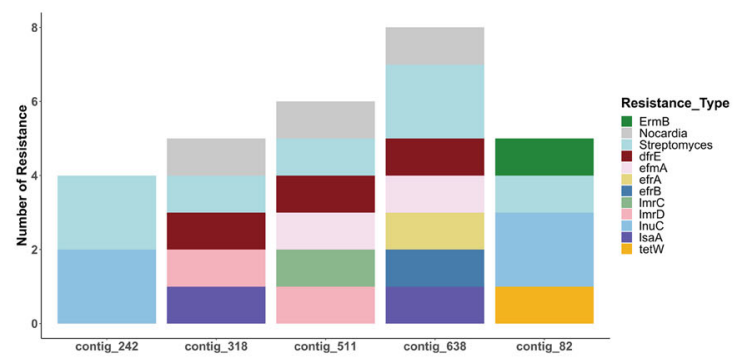

b

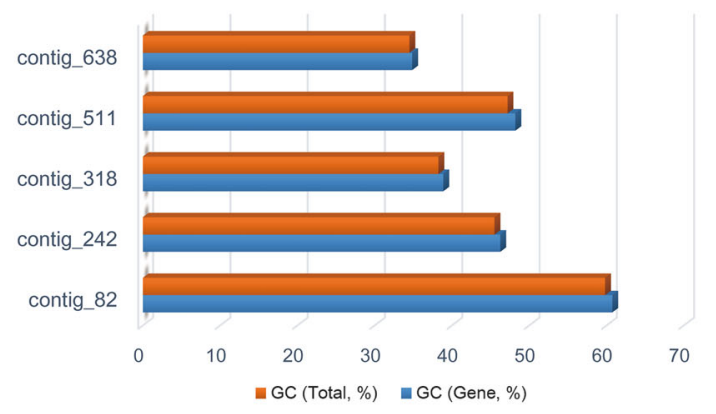

d

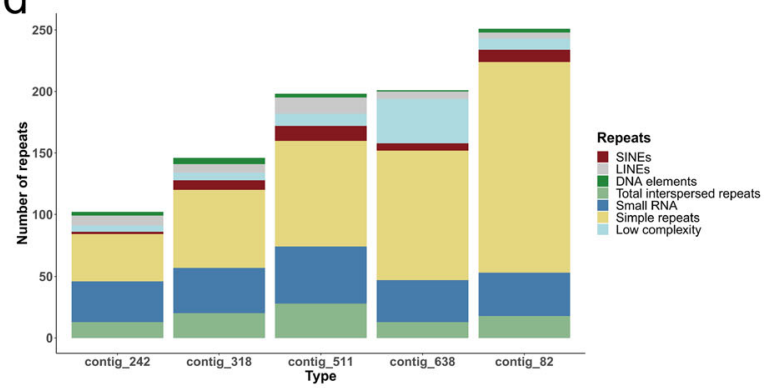

f

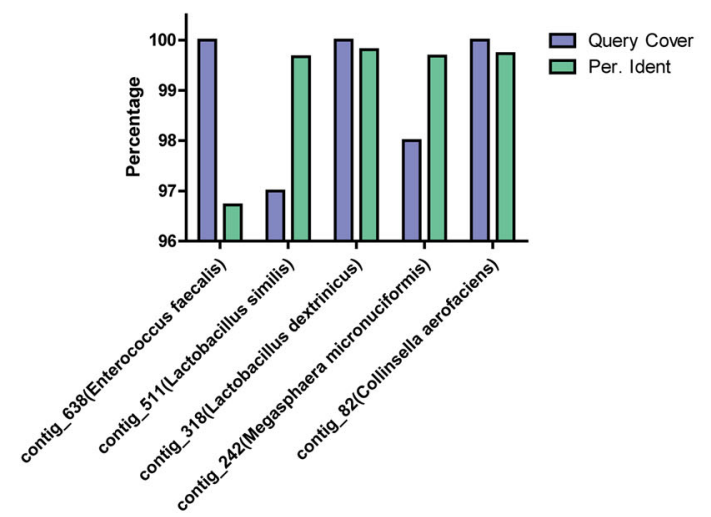

Fig. 3 The genome analysis of the five non-match genomes. a The genome length and gene number of the five non-match genomes. $\mathbf{b}$ The GC content(total and gene) of the contigs in the two samples of the five genomes. $\mathbf{c}$ The ncRNA analysis (rRNA, tRNA and sRNA) of the five genomes. d The repeat sequences of the five genomes. e The antibiotic potential of the five genomes. $\mathbf{f}$ The blasted result of the full-length $16 \mathrm{~S}$ rRNAs of the five sequences in the NCBI database. The reference bacteria are shown in parentheses.

in Tongji University, we named it Enterococcus tongjius. We then analyzed the function potential of the genome and found that many genes might be correlated with carbohydrate and lipid metabolism (Fig. 5e). Interestingly, the function potential of many genes was not clear (Fig. 5e). Further analysis of sugar metabolism showed that most genes were associated with hydrolysis (Fig. 5f). Finally, we analyzed the whole genome of Enterococcus tongiius and found it contains a total of $2.1 \mathrm{M}$ bases, and the GC content is relatively low $(34.49 \%)$, which is also consistent with other species of Enterococci (Fig. 5g). It had 2111 genes, 12 rRNAs, and 63 tRNAs (Fig. 5g).

\section{Discussion}

Extensive studies of the human gut microbiota have been reported in past decades. However, large numbers of species in the gut microbiota remain unknown. By improving the extraction method, we successfully acquired high-quality genomic DNA of the gut microbiota, and obtained approximately 85 Gb TGS data from 
a

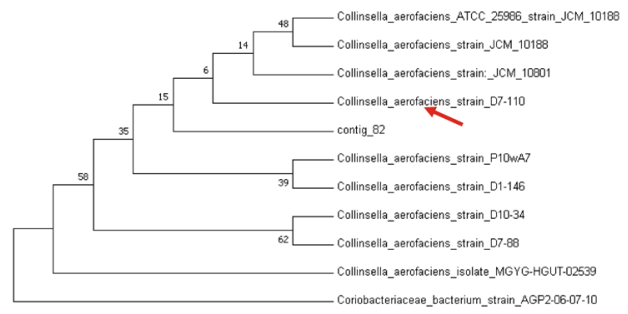

C

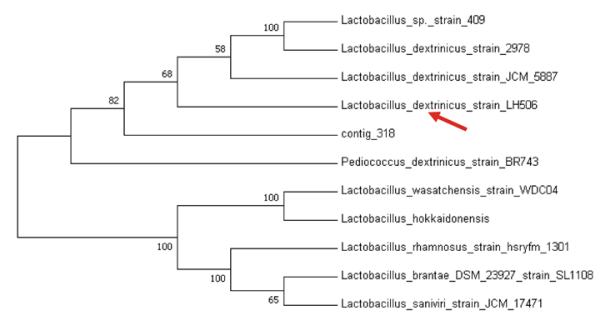

b

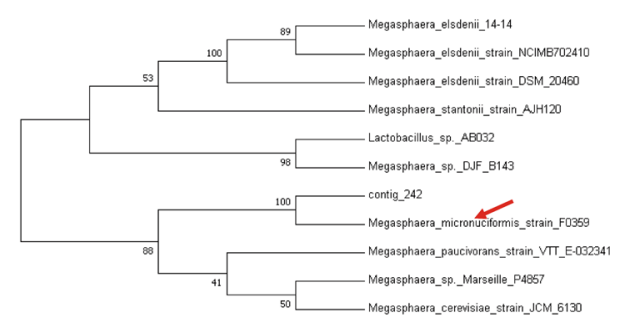

d

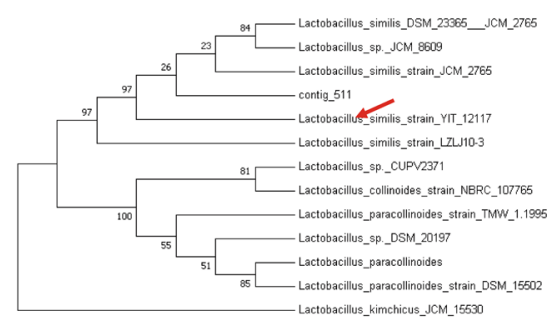

e

contig 82

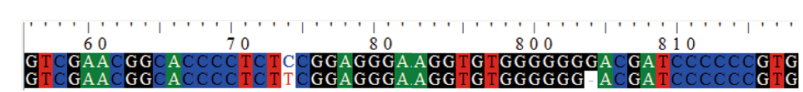

Collinsella aerofaciens strain D7-110

contig 242

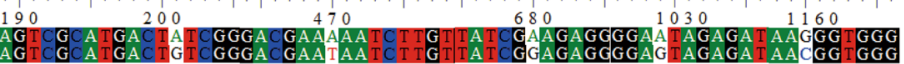

contig 318
Lactobacillus dextrinicus strain LH506

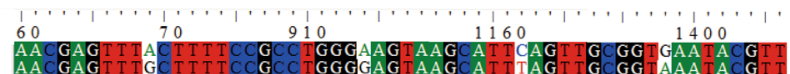

contig 511
Lactobacillus similis strain YIT 12117

f

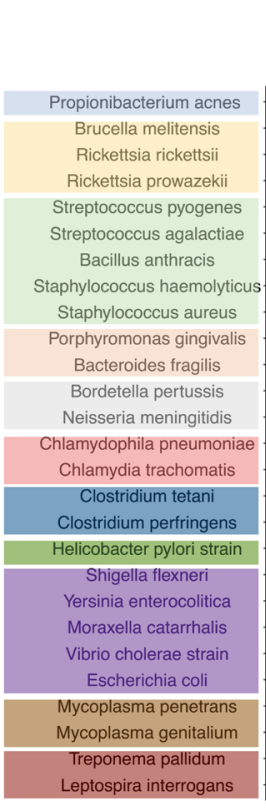

Identity (\%)

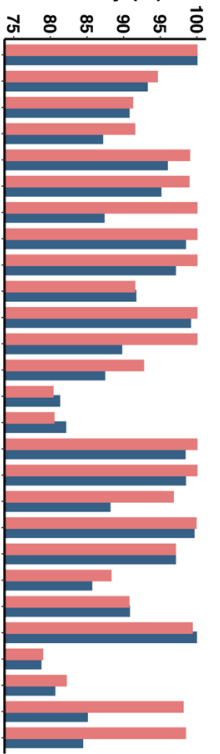

Lignment Length

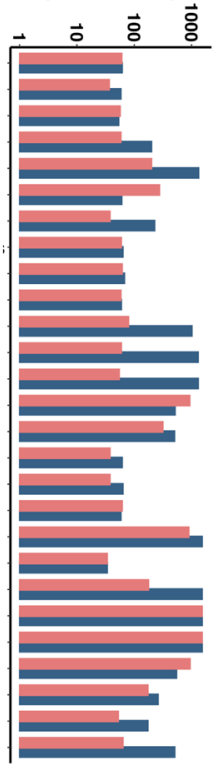

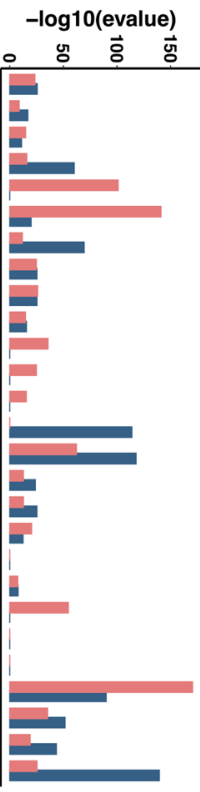

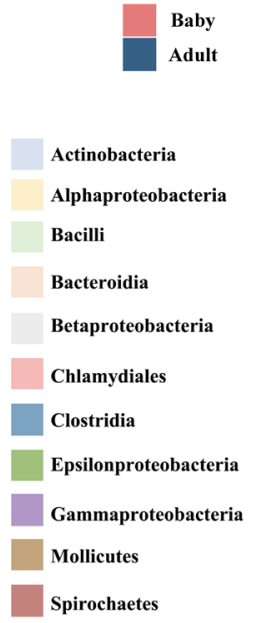

Fig. 4 Phylogeny of four high identity non-match genomes. a-d Phylogenetic tree of the high identity non-match genomes through full-length $16 \mathrm{~s}$ rRNAs, showing their accurate taxonomic location. e ClustalW multiple alignment of the full-length $16 \mathrm{~s}$ rRNAs of these genomes with that of the related bacterial strains. $\mathbf{f}$ Conditional pathogens Shigella flexneri lived in the gut of adult and baby. 
a

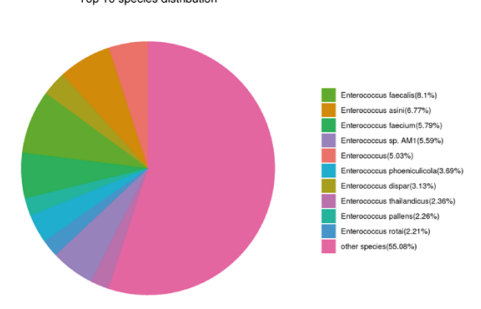

C
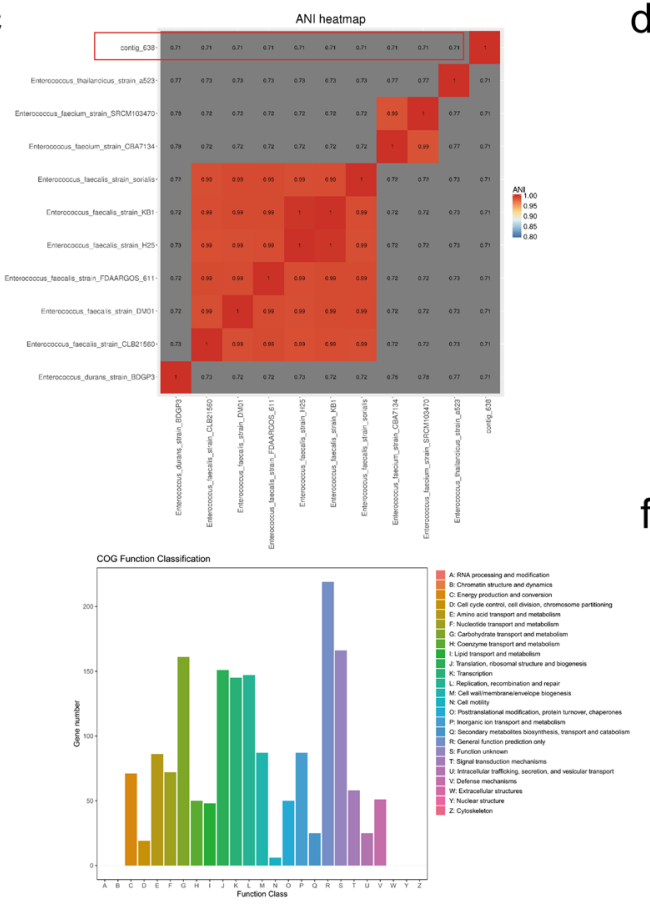

b

d
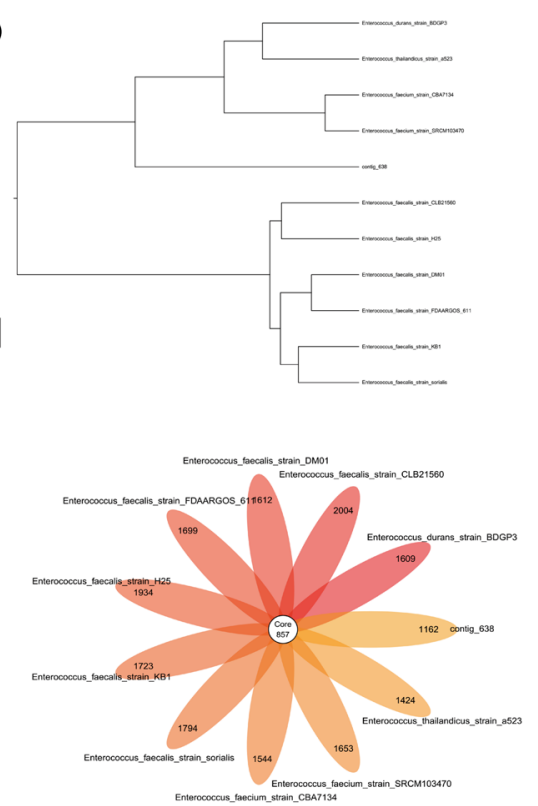

f

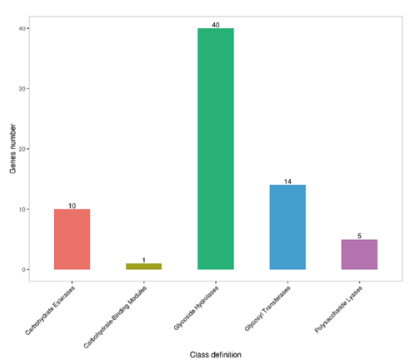

\section{g}
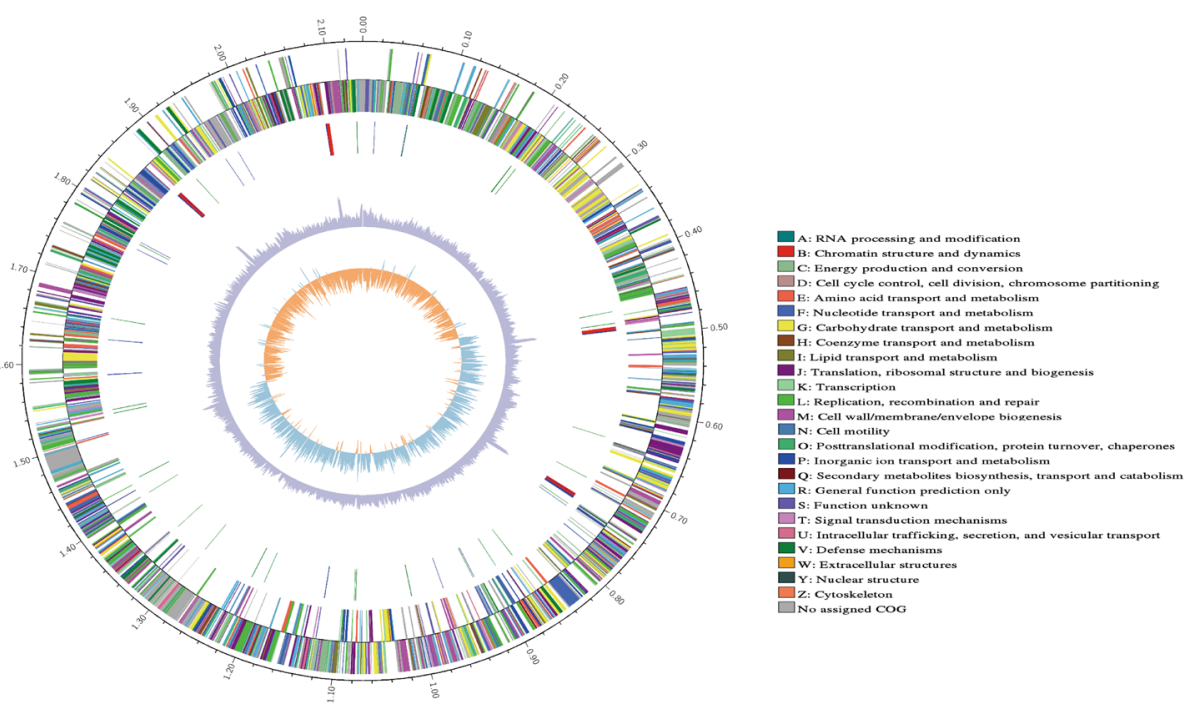

Fig. 5 Genome aspects of the new species of Enterococcus tongjius. a Non-redundant database annotations of contig_638. b Phylogenetic tree of the high contig_638 through whole genomes. c The ANI analyses showed that contig_638 was a total new species since the ANI score was only 0.71, much lower than 0.9. The red frame displayed the scores of contig_638. $\mathbf{d}$ A flower-plot schematic representation illustrates the number of predicted core (857) genes and distinctive genes. e Function analyses of the CDSs of the Enterococcus tongjius. $\mathbf{f}$ Carbohydrate-active enzymes analysis of the Enterococcus tongjius. g Circos diagram of the whole genome of the Enterococcus tongjius (From the inner to the outer are GC skew, GC content, non-coding RNA (rRNA red, tRNA blue, sRNA green), lagging strand COG annotation, leading strand COG annotation). 
each sample, which was much greater than previous studies $^{23,34,35}$. We successfully assembled nine bacterial genomes and large numbers of contigs using this method. Interestingly, more than $50 \%$ of the genomes (five of nine bacterial genomes) might be new human species or subspecies. Using large-scale metagenomic assembling, Edoardo et al. and Alexandre et al. uncovered thousands of new species of bacteria ${ }^{9,36}$. These results, together with our work, indicated that further investigation of gut microbiota is worthwhile. Furthermore, the approach used in this study will help assemble the genome of unknown bacteria, and thus might facilitate the Human Microbiome Project and the Genomic Encyclopedia of Bacteria and Archaea ${ }^{37,38}$. Finally, bacteria are widely distributed in various environments, such as natural lakes, oceans, and soils. Therefore, it is of great interest to investigate bacteria living in different environments with our methods.

Compared with the metagenome, genes in the microbial genome have traditionally been underestimated. For example, Hila et al. found that there are a fairly large number of unknown small proteins in the human microbiome ${ }^{39}$. Intriguingly, our work discovered more than 10,000 unknown genes without known domain (Fig. 1d). These findings indicated that a vast number of unidentified genes are needed to be explored in the human microbiome. Importantly, some bacterial genes are valuable for human health. For example, an anti-inflammatory protein from Faecalibacterium prausnitzii could inhibit the NF- $\kappa B$ pathway in intestinal epithelial cells $^{40}$. Via KEGG analysis, we found that most genes in the two samples were closely associated with human basic physiological activities (Supplementary Fig. 3a, b). In addition, bacterial genes are potential disease diagnostic indicators because they can pass through intestinal epithelial cells and enter the plasma ${ }^{41}$. The exploration of drugmetabolizing enzymes from the gut microbiota may be useful in drug development and personalized medicine $^{7,42}$. Our research will help discover bacterial proteins linking human health and diseases.

In the nine assemble genomes, we discovered a new bacterium, Enterococcus tongjium. We discovered that it belongs to Enterococcus, a ubiquitous Gram-positive genus with low-GC genomes. Many members of this genus are pathogenic bacteria or conditional pathogens, because of their role as primary causative agents of healthcare-associated infections ${ }^{43,44}$. Therefore, this bacterium might be a conditional pathogen. We also found two other conditional pathogens: Megasphaera micronuciforrnis and Shigella flexneri. An important question remains: what matters to human health: the quality or quantity of microbes ${ }^{45}$ ? The results in this work suggested that the quality and quantity of microbes were both important for our health.
Despite the promise that this study holds for gut microbiota, it is important to note its limitations. First, the lengths of bacterial genomes acquired were between 1.5 and $3.5 \mathrm{Mb}$, much smaller than that of E. coli str. K-12 (4.64 Mb). We found that the contig lengths were closely correlated with the coverage (Supplementary Fig. 5). Therefore, it is essential to improve the data quantity of each sample to obtain longer contigs. With the continuous development of TGS technology, it is probable that more sequencing data can be acquired in a single sample. Second, although we established an effective method, it is still not easy to acquire sufficient high quality genomic DNA, and we only harvested two samples. It is urgent to improve these methods and to analyze more human fecal samples. At last, we also tried to cultivate the newly identified species, Enterococcus tongjius, with multiple traditional microbiological means, including different medium and antibiotics; but we have not isolated Enterococcus tongjius clone successfully until now. Our inability to culture this bacterium has limited our understanding of its ecological role in the intestinal environment and its relation to human's health.

In summary, we established an effective extraction method to obtain high quality gut microbiota genomic DNA and detected the samples with TGS technology. We identified not only a large number of unknown genes, but also several new subspecies and species with our methods. This work provided a novel and reliable framework for exploring gut microbiota genomes, improving our understanding of the mechanisms that underlie the role of the microbiome in health and disease.

\section{Acknowledgements}

This work was supported by Special clinical research project of Shanghai Municipal Health Commission (20194Y0458); Pandeng Plan of Shanghai

University of Medicine and Health Sciences (B3-0200-20-311007-6); Academic Mentorship for Scientific Research Cadre Project (AMSCP-21-06); China Postdoctoral Science Foundation funded project (2018M632169); Shanghai Medical Key Specialty Construction Fund (ZK2019B25); Key Disciplines Group Construction Project of Pudong Health Bureau of Shanghai(PWZxq2017-01); Outstanding Clinical Discipline Project of Shanghai Pudong (PWYgy2018-03).

\section{Author details \\ 'Shanghai University of Medicine \& Health Sciences affiliated Zhoupu Hospital, 201318 Shanghai, China. ${ }^{2}$ School of Basic Medical Sciences and Shanghai Key Laboratory of Molecular Imaging, Shanghai University of Medicine and Health Sciences, 201318 Shanghai, China. ${ }^{3}$ Shanghai OE Biotech Company, 201114 Shanghai, China. ${ }^{4}$ Shanghai First Maternity and Infant Hospital, Tongji University School of Medicine, 201204 Shanghai, China. ${ }^{5}$ Shanghai East Hospital, Tongji University School of Medicine, 200120 Shanghai, China}

\section{Author contributions}

J.H. and J.S. conceived and designed the research. Y.L. and J.S. collected samples and extracted the bacteria genome DNA. J.Z. sequenced DNA and assembled the contigs. J.Z. and J.H. analyzed and interpreted the data. Y.J., H.P., L.W., D.L., and J.L. performed microbiome analysis. Y.L, J.H., and J.S. wrote and revised the manuscript. All authors have read and approved the final manuscript.

\section{Data availability}

The raw sequencing data generated from this study have been deposited in NCBI SRA (https://www.ncbi.nlm.nih.gov/sra) under the accession number PRJNA717332. 


\section{Conflict of interest}

The authors declare no competing interests.

\section{Publisher's note}

Springer Nature remains neutral with regard to jurisdictional claims in published maps and institutional affiliations.

Supplementary information The online version contains supplementary material available at https://doi.org/10.1038/s41419-021-03829-y.

Received: 2 December 2020 Revised: 11 May 2021 Accepted: 12 May 2021 Published online: 02 June 2021

\section{References}

1. Dominguez-Bello, M. G., Godoy-Vitorino, F., Knight, R. \& Blaser, M. J. Role of the microbiome in human development. Gut 68, 1108-1114 (2019).

2. Visconti, A. et al. Interplay between the human gut microbiome and host metabolism. Nat. Commun. 10, 4505 (2019).

3. D'Amelio, P. \& Sassi, F. Gut microbiota, immune system, and bone. Calcif. Tissue Int. 102, 415-425 (2018).

4. Gopalakrishnan, V., Helmink, B. A., Spencer, C. N., Reuben, A. \& Wargo, J. A. The influence of the gut microbiome on cancer, immunity, and cancer immunotherapy. Cancer Cell 33, 570-580 (2018).

5. $\mathrm{Ma}, \mathrm{C}$. et al. Gut microbiome-mediated bile acid metabolism regulates liver cancer via NKT cells. Science 360, eaan5931 (2018).

6. Zhu, S. B. et al. The progress of gut microbiome research related to brain disorders. J. Neuroinflamm. 17, 25 (2020).

7. Zimmermann, M., Zimmermann-Kogadeeva, M., Wegmann, R. \& Goodman, A. L. Mapping human microbiome drug metabolism by gut bacteria and their genes. Nature 570, 462-467 (2019).

8. Javdan, B. et al. Personalized mapping of drug metabolism by the human gut microbiome. Cell 181, 1661-1679 (2020).

9. Pasolli, E. et al. Extensive unexplored human microbiome diversity revealed by over 150,000 genomes from metagenomes spanning age, geography, and lifestyle. Cell 176, 649-662 (2019).

10. Quince, C., Walker, A. W., Simpson, J. T., Loman, N. J. \& Segata, N. Shotgun metagenomics, from sampling to analysis. Nat. Biotechnol. 35, 833-844 (2017).

11. Diwan, V., Albrechtsen, H. J., Smets, B. F. \& Dechesne, A. Does universal 165 rRNA gene amplicon sequencing of environmental communities provide an accurate description of nitrifying guilds? J. Microbiol. Methods 151, 28-34 (2018).

12. Johnson, J. S. et al. Evaluation of $16 \mathrm{~S}$ rRNA gene sequencing for species and strain-level microbiome analysis. Nat. Commun. 10, 5029 (2019).

13. Poretsky, R., Rodriguez-R, L. M., Luo, C. W., Tsementzi, D. \& Konstantinidis, K. T. Strengths and limitations of 165 rRNA gene amplicon sequencing in revealing temporal microbial community dynamics. PLoS ONE 9, e93827 (2014).

14. Laudadio, I. et al. Quantitative assessment of shotgun metagenomics and $16 \mathrm{~S}$ rDNA amplicon sequencing in the study of human gut microbiome. OMICS 22, 248-254 (2018).

15. Ranjan, R., Rani, A., Metwally, A., McGee, H. S. \& Perkins, D. L. Analysis of the microbiome: advantages of whole genome shotgun versus $16 \mathrm{~S}$ amplicon sequencing. Biochem Biophys. Res. Commun. 469, 967-977 (2016).

16. Stewart, R. D. et al. Assembly of 913 microbial genomes from metagenomic sequencing of the cow rumen. Nat. Commun. 9, 870 (2018).

17. Somerville, V. et al. Long-read based de novo assembly of low-complexity metagenome samples results in finished genomes and reveals insights into strain diversity and an active phage system. BMC Microbiol. 19, 143 (2019).

18. Abdullah, W. Z. W., Mackey, B. M. \& Karatzas, K. A. G. High phenotypic variability among representative strains of common Salmonella enterica serovars with possible implications for food safety. J. Food Prot. 81, 93-104 (2018).
19. Knopp, M. \& Andersson, D. I. Predictable phenotypes of antibiotic resistance mutations. Mbio 9, e00770-18 (2018).

20. van Dijk, E. L., Jaszczyszyn, Y., Naquin, D. \& Thermes, C. The third revolution in sequencing Technology. Trends Genet. 34, 666-681 (2018).

21. Ge, H. et al. De novo assembly of a chromosome-level reference genome of red-spotted grouper (Epinephelus akaara) using nanopore sequencing and $\mathrm{Hi}$ C. Mol. Ecol. Resour. 19, 1461-1469 (2019).

22. Holm, J. B. et al. Comparative metagenome-assembled genome analysis of "Lachnocurva vaginae", formerly Known as bacterial vaginosis-associated bacterium-1 (BVAB1). Front. Cell. Infect. Microbiol. 10, 117 (2020).

23. Wongsurawat, T. et al. An assessment of Oxford Nanopore sequencing for human gut metagenome profiling: a pilot study of head and neck cancer patients. J. Microbiol. Methods 166, 105739 (2019).

24. Low, W. Y. et al. Chromosome-level assembly of the water buffalo genome surpasses human and goat genomes in sequence contiguity. Nat. Commun. 10, 260 (2019).

25. Kumar, S., Stecher, G. \& Tamura, K. MEGA7: molecular evolutionary genetics analysis version 7.0 for bigger datasets. Mol. Biol. Evol. 33, 1870-1874 (2016).

26. Kim, M., Oh, H. S., Park, S. C. \& Chun, J. Towards a taxonomic coherence between average nucleotide identity and 16S rRNA gene sequence similarity for species demarcation of prokaryotes. Int. J. Syst. Evol. Microbiol. 64, 346-351 (2014).

27. Hyatt, D. et al. Prodigal: prokaryotic gene recognition and translation initiation site identification. BMC Bioinform. 11, 119 (2010).

28. Lowe, T. M. \& Eddy, S. R. tRNAscan-SE: a program for improved detection of transfer RNA genes in genomic sequence. Nucleic Acids Res. 25, 955-964 (1997).

29. Lagesen, K. et al. RNAmmer: consistent and rapid annotation of ribosomal RNA genes. Nucleic Acids Res. 35, 3100-3108 (2007).

30. Griffiths-Jones, S., Bateman, A., Marshall, M., Khanna, A. \& Eddy, S. R. Rfam: an RNA family database. Nucleic Acids Res. 31, 439-441 (2003).

31. Tarailo-Graovac, M. \& Chen, N. Using RepeatMasker to identify repetitive elements in genomic sequences. Curr. Protoc. Bioinform. Chapter 4, 10 (2009).

32. Jia, B. et al. CARD 2017: expansion and model-centric curation of the comprehensive antibiotic resistance database. Nucleic Acids Res. 45, D566-D573 (2017).

33. Cantarel, B. L. et al. The carbohydrate-active EnZymes database (CAZy): an expert resource for glycogenomics. Nucleic Acids Res. 37, D233-D238 (2009).

34. Suzuki, Y. et al. Long-read metagenomic exploration of extrachromosomal mobile genetic elements in the human gut. Microbiome 7, 119 (2019).

35. Song, W. Z., Thomas, T. \& Edwards, R. J. Complete genome sequences of pooled genomic DNA from 10 marine bacteria using PacBio long-read sequencing. Mar. Genomics 48, 35-43 (2019).

36. Almeida, A. et al. A new genomic blueprint of the human gut microbiota. Nature 568, 499-504 (2019).

37. Mukherjee, S. et al. 1003 reference genomes of bacterial and archaeal isolates expand coverage of the tree of life (vol 35, pg 676, 2017). Nat. Biotechnol. 36, 368-368 (2018).

38. Proctor, L. M. et al. The integrative human microbiome project. Nature $\mathbf{5 6 9}$, 641-648 (2019)

39. Sberro, H. et al. Large-scale analyses of human microbiomes reveal thousands of small, novel genes. Cell 178, 1245-1259 (2019).

40. Quevrain, E. et al. Identification of an anti-inflammatory protein from Faecalibacterium prausnitzii, a commensal bacterium deficient in Crohn's disease. Gut 65, 415-425 (2016).

41. Abasiyanik, M. F. et al. Ultrasensitive digital quantification of cytokines and bacteria predicts septic shock outcomes. Nat. Commun. 11, 2607 (2020).

42. Zimmermann, M., Zimmermann-Kogadeeva, M., Wegmann, R. \& Goodman, A. L. Separating host and microbiome contributions to drug pharmacokinetics and toxicity. Science 363, eaat9931 (2019).

43. Ben Braiek, O. \& Smaoui, S. Enterococci: between emerging pathogens and potential probiotics. Biomed. Res. Int. 2019, 5938210 (2019).

44. Garcia-Solache, M. \& Rice, L. B. The enterococcus: a model of adaptability to its environment. Clin. Microbiol. Rev. 32, e00058-18 (2019).

45. Cani, P. D. Human gut microbiome: hopes, threats and promises. Gut 67, 1716-1725 (2018) 